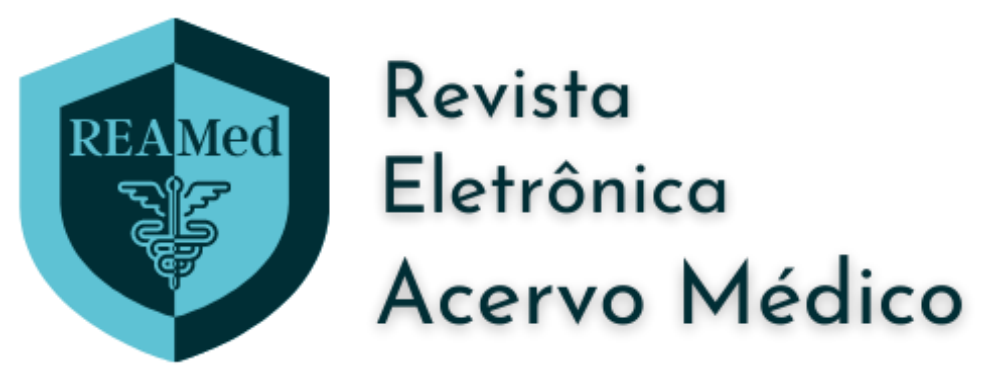

\title{
Tabagismo no Brasil: impacto econômico na saúde pública e seu tratamento
}

\author{
Smoking in Brazil: economic impact on public health and its treatment
}

Tabaquismo en Brasil: impacto económico en la salud pública y su tratamiento

Charles Bernardo Buteri Filho ${ }^{1}$, Marcus Vinícius Monteiro Martins ${ }^{1 \star}$, Larissa Zolini Gomes ${ }^{2}$, Kalyne Maria de Paiva Nalon², Vinícius Barcelos de Carvalho ${ }^{3}$, Raissa Chades Pinheiro Fonseca ${ }^{4}$, Thiago Leister de Medeiros ${ }^{1}$, Charles Bernardo Buteri ${ }^{5}$.

\section{RESUMO}

Objetivo: Demonstrar os efeitos do tabagismo no Brasil, no tocante da saúde pública e na economia. Revisão bibliográfica: $O$ tabagismo é um problema de saúde pública, causa mortalidade, morbidade e gera custos para o sistema. O Brasil apesar de ser referência internacional no assunto, ainda apresenta grande número absoluto de tabagista e sofre de suas consequências. Por essa razão, é importante atuar no tratamento desse vício. Sabe-se que a melhora forma de tratamento é a combinação entre medidas farmacológicas e não farmacológicas. E essas medidas podem ser empregadas de diferentes maneiras e com diferentes graus de evidência e eficácia. Devendo, cada abordagem ser individualizada para cada perfil de paciente. Considerações finais: Entender o contexto do tabagismo no país é fundamental para tomar dimensão dos efeitos do problema. Ao tomar tal consciência, fica clara, a importância do tratamento do tabagismo. O qual pode ser empregado de muitas formas, necessitando para isso de um bom conhecimento básico do assunto.

Palavras-chave: Tabagismo, Saúde pública, Tratamento no Brasil.

\begin{abstract}
Objective: To demonstrate the effects of smoking in Brazil, in terms of public health and the economy. Bibliographic review: Smoking is a public health problem, causes mortality, morbidity and generates costs for the system. Brazil, despite being an international reference on the subject, still has a large absolute number of smokers and suffers from its consequences. For this reason, it is important to act in the treatment of this addiction. It is known that the best form of treatment is the combination of pharmacological and nonpharmacological measures. And these measures can be used in different ways and with different degrees of evidence and effectiveness. Therefore, each approach must be individualized for each patient profile. Final considerations: Understanding the context of smoking in the country is essential to understand the effects of
\end{abstract}

\footnotetext{
${ }^{1}$ Universidade Federal de Ouro Preto (UFOP), Ouro Preto - MG. Email: marcusufla@gmail.com

2 Universidade Federal de Lavras (UFLA), Lavras - MG.

3 Prefeitura Municipal de Ouro Preto, Ouro Preto - MG.

${ }^{4}$ Hospital Metropolitano Odilon Behrens, Belo Horizonte - MG.

5 Instituto Federal de Educação, Ciência e Tecnologia do Norte de Minas (IFNMG), Salinas - MG.
} 
the problem. Upon becoming aware of this, the importance of treating smoking becomes clear. Which can be used in many ways, requiring a good basic knowledge of the subject.

Keywords: Smoking, Public health, Treatment in Brazil.

\section{RESUMEN}

Objetivo: Demostrar los efectos del tabaquismo en Brasil, en términos de salud pública y economía. Revisión bibliográfica: El tabaquismo es un problema de salud pública, causa mortalidad, morbilidad y genera costos para el sistema. Brasil, a pesar de ser un referente internacional en el tema, todavía tiene un gran número absoluto de fumadores y sufre sus consecuencias. Por ello, es importante actuar en el tratamiento de esta adicción. Se sabe que la mejor forma de tratamiento es la combinación de medidas farmacológicas y no farmacológicas. Y estas medidas pueden usarse de diferentes formas y con diferentes grados de evidencia y efectividad. Por lo tanto, cada abordaje debe individualizarse para cada perfil de paciente. Consideraciones finales: Comprender el contexto del tabaquismo en el país es fundamental para comprender los efectos del problema. Al tomar conciencia de esto, queda clara la importancia de tratar el tabaquismo. Que se puede utilizar de muchas formas, requiriendo un buen conocimiento básico del tema.

Palabras clave: Tabaquismo, Salud pública, Tratamiento en Brasil.

\section{INTRODUÇÃO}

O tabagismo é definido pela Organização Mundial da Saúde (OMS), como uma doença crônica. Sendo responsável pela morte de cerca de 8 milhões de pessoas no mundo, além de causar grande morbidade (SZKLO AS e BERTONI N, 2020).

No Brasil, o tabagismo tem diminuído e segundo dados da Pesquisa Nacional de Saúde (PNS), a parcela de fumantes adultos no Brasil foi de 12,6\% uma queda percentual de $63,8 \%$ nesses últimos 30 anos (INSTITUTO NACIONAL DO CÂNCER (INCA), 2021a).

Sendo o Brasil, referência internacional no combate ao fumo (PORTES LH, et al., 2018). Porém, apesar disso, o tabagismo ainda exerce grande impacto econômico para o Brasil, quando se pensa nos gastos gerados para a saúde pública. Assim, o tabagismo gera problemas em diferentes vertentes, justificando a relevância do seu tratamento (INCA, 2018).

O tratamento ideal é a combinação de medidas comportamentais e tratamento farmacológico. Podendo ser feito de várias maneiras. Assim, é esperado ao profissional de saúde o entendimento básico do assunto. Por isso, a importância de revisar tal assunto, como proposto nesta revisão (WINDLE SB, et al., 2016).

O objetivo deste estudo foi demonstrar os efeitos do tabagismo no Brasil, no tocante da saúde pública e na economia.

\section{REVISÃO BIBLIOGRÁFICA}

\section{Histórico do tabagismo}

O tabaco surgiu na América pré-colonial. No século XVI, foi levado para a Europa por navegadores e de lá se espalhou para todo o resto do mundo. Na metade do século XIX o cigarro industrializado se popularizou e esse passou a ser produzido em larga escala favorecendo a expansão do consumo mundial do tabaco (INCA, 2012). Porém, hoje, o tabagismo é tido como uma doença crônica e o tabagismo guarda causalidade com cerca de 50 doenças incapacitantes e fatais (OMS, 2020)

O Brasil continua como o segundo colocado no ranking dos países produtores de fumo em folha (INCA, 2020). Contudo, apesar de ser um dos maiores exportadores de fumo do mundo, o Brasil também se apresenta como referência internacional no controle do uso do tabaco (PORTES LH, et al., 2018). Em 2019, 
segundo dados da PNS, a parcela de fumantes adultos no Brasil foi de $12,6 \%$ uma queda percentual de $63,8 \%$ nesses últimos 30 anos. Logo, estima-se que cerca de 420.000 mortes, relacionadas ao tabaco, foram evitadas (INCA, 2021a).

\section{Impacto econômico}

Em 2019 o tabaco ocupou o $22^{\circ}$ lugar no ranking dos produtos exportados pelo país, com um faturamento de $\mathrm{R} \$ 6,28$ bilhões na sua produção. No entanto, em relação aos custos atribuíveis ao tabagismo, em 2015, o consumo de tabaco gerou cerca de 40 bilhões de reais em gastos em assistência médica, o que representou $8 \%$ do total de gasto em saúde no ano. Acrescentando-se a este valor os custos indiretos, representados pela perda de produtividade, pela incapacidade ou morte prematura dos fumantes, estimou-se um gasto adicional de outros 17 bilhões de reais, elevando o valor para 57 bilhões de reais atribuídos ao tabagismo. Contudo, os impostos sobre a venda dos produtos do tabaco arrecadaram em 2015, aproximadamente 13 bilhões de reais, o que correspondeu a $32,5 \%$ dos gastos diretos e a $22,8 \%$ do total de gasto, o que deixa o país com um prejuízo, estimado de 44 bilhões de reais, o que representa aproximadamente $0,79 \%$ do Produto Interno Bruto de 2015 (SINDICATO INTERESTADUAL DA INDÚSTRIA DO TABACO (SINDITABACO), 2019).

Nesse contexto, em 2020, uma publicação do Instituto de Efetividade Clínica e Sanitária estimou os gastos totais atribuíveis ao tabagismo em 125,2 bilhões ao ano, isso sem incluir as ações de prevenção e tratamento para cessação do consumo, nem os custos com o controle do mercado ilegal do tabaco. Ainda, do total atribuído ao Tabagismo no ano de 2020: 50,3 bilhões foram gastos com custos médicos diretos, o que equivale a $7,8 \%$ do total gasto em saúde no ano. Sendo que, o total de custos atribuíveis ao tabagismo em 2020 correspondeu a 1,69\% do PIB brasileiro, segundo os dados do Instituto Brasileiro de Geografia e Estatística. Assim, o total arrecadado pelos impostos sobre a venda do tabaco e seus derivados em 2020, não cobre os custos econômicos totais desencadeados pelo tabagismo (INCA, 2021b).

\section{Saúde pública}

A principal causa de mortes prematuras evitáveis no mundo é o tabagismo. A doença mata anualmente perto de 8 milhões de pessoas no mundo e foi responsável pela morte de aproximadamente 100 milhões de pessoas no século XXI. No Brasil é responsável por aproximadamente 156 mil mortes por ano, além de gerar morbidade e, tanto o tabagismo ativo quanto na exposição passiva, à fumaça do cigarro se relacionam com o aparecimento de enfermidades (SZKLO AS e BERTONI N, 2020). Dentre estas, vários tipos de cânceres e doenças do aparelho respiratório, doenças cardiovasculares, complicações na gravidez, impotência sexual, catarata, patologias da boca, úlcera do aparelho digestivo e osteoporose (INCA, 2021c). Sendo algunsdos fatores que se relacionam com uma maior prevalência do consumo são: condição socioeconômica desfavorável e de menor escolaridade (ROCHA SAV, et al., 2019).

Ademais, no contexto atual de pandemia, estudos indicam desfechos piores da COVID-19 em pacientes tabagistas e que ainda o ato de fumar amplia os riscos relacionados ao contágio (INCA, 2021d). Na infecção pela COVID-19 as chances de complicação em fumantes é $45 \%$ maior, e a mortalidade aumenta em 38\%, já naqueles fumantes que já possuem a doença pulmonar obstrutiva crônica, o risco de complicações quando comparado aos não fumantes é de $88 \%$ a mais e também foi observado, neste grupo, o aumento do risco de morte em $60 \%$. Assim, se o tabaco fosse uma droga farmacêutica, ele jamais seria licenciado e nem sua venda lícita permitida (ALQAHTANI JS, 2020)..

\section{Tratamento}

O tratamento do tabagismo deve ser de fácil acesso, acolhedor e levar em conta o contexto social. De modo que, segundo a OMS, $60 \%$ dos tabagistas do mundo desejam parar de fumar, mas apenas 30\% têm acesso a serviços apropriados (INCA, 2021e).

Existem diversas estratégias e guias de recomendações para auxiliar na cessação do tabagismo. Porém, os pontos em comum são que essas abordagens trabalham conceitos de dependência química, questões psicológicas, comportamentais e sociais. Portanto, múltiplos fatores influenciam na cessação do tabagismo. Doenças psiquiátricas, baixa motivação, sedentarismo, ausência de doença relacionada ao tabaco, início do consumo na vida adulta, tabagistas com menos de 20 anos de uso, ser solteiro, ter maior escolaridade, não 
participar das sessões de manutenção da abstinência e presença de gatilhos, podem ser fatores que influenciam negativamente (MINISTÉRIO DA SAÚDE, 2020)

Em relação às formas de parar de fumar, os métodos utilizados são: a parada abrupta e o a redução gradual, esse com maior eficácia. Nesse último, o tabagista reduz o número de cigarros a cada dia até parar, ou adia o horário do primeiro cigarro progressivamente até parar. Sendo que a redução gradual não deve levar mais de duas semanas até a cessação (MACIEL RDR, et al., 2021).

No entanto, para que o sucesso seja obtido à avaliação do grau de motivação do fumante é essencial e para avalia-la, a escala mais usada é a de Proschaska e colaboradores que classifica em estágios. Précontemplação, quando o indivíduo mesmo conhecendo os benefícios de parar de fumar não cogita essa possibilidade. Contemplação, no qual apesar de admitir o problema, ainda evita parar de fumar. E, por fim, preparação, fase na qual os primeiros passos da cessação são dados. E dentro desse último, há a fase de ação, na qual a pessoa adota atitudes que a levam efetivamente a parar de fumar e a fase de manutenção, onde, após a cessação do hábito, o foco está em manter a mudança, configurando a finalização do processo (RIGOTTI NA, et al., 2021).

Nessa lógica, uma das técnicas utilizadas para promover a cessação do tabagismo é a terapia cognitivocomportamental, que entende a dependência como um elemento de comportamento aprendido e modificável. Portanto, o foco da Terapia Cognitivo-Comportamental é na mudança de crenças e comportamentos (LOPES FM e SILVEIRA KM, 2020).

Além disso, recomenda-se que todo profissional de saúde faça pelo menos a intervenção/aconselhamento breve aos pacientes tabagistas, independente do atendimento. Nessa abordagem, pode ser adotada a intervenção breve/mínima, que consiste em realizar perguntas para avaliar, aconselhar e preparar o tabagista, sem acompanhá-lo, com duração de três a cinco minutos, podendo ser realizada em qualquer contato com o paciente e por qualquer profissional de saúde (SANTOS MDV, et al., 2019).

Por isso, toda a equipe deveria ser treinada para realizar algumas perguntas que levem o paciente a tentar parar de fumar, como: Você fuma? Há quanto tempo? Quantos cigarros por dia? Em quanto tempo após acordar acende o primeiro cigarro? O que você acha de marcar uma data para parar de fumar? Quando? Já tentou parar? O que aconteceu? Assim, as perguntas têm como foco principal diferenciar a experimentação do uso regular; avaliar as chances do paciente desenvolver sintomas de abstinência; descobrir em qual estágio de motivação o paciente se encontra e identificar pontos forte e fracos nas tentativas anteriores de cessação (SANTOS MDV, et al., 2019).

De forma que, embora a abordagem breve/mínima não seja a forma ideal de atendimento, essa tem a capacidade de aumentar as chances de cessação quando comparadas a nenhum aconselhamento. Também pode ser adotada a abordagem básica, que é mais recomendada que a abordagem breve/mínima. Podendo ser feita por qualquer profissional de saúde, com duração de 3 a 5 minutos. Com a diferença que prevê consultas de seguimento, para auxiliar melhor na fase de abstinência. Nessa abordagem, os tabagistas que não estiverem dispostos a parar em até 30 dias devem ser incentivados e abordados em uma próxima oportunidade. Já os interessados devem ser incentivados a marcar uma data para a cessação, esclarecidos quanto aos sintomas de abstinência, estratégias para se manter abstinente, evitar gatilhos e se desfazer de cigarros e isqueiros (MINISTÉRIO DA SAÚDE 2020).

Ademais, a entrevista motivacional é uma técnica que auxilia na mudança do estágio de motivação em que o paciente se encontra. Para realizá-la o profissional deve acolher o fumante e compreender seus conflitos em relação ao tabagismo, analisar os elementos que impedem a mudança, minimizar as incertezas do tabagista e enfatizar os benefícios que serão alcançados com a abstinência. Entretanto, nas pesquisas realizadas, tanto a entrevista motivacional quanto a abordagem breve não tiveram resultados satisfatórios, principalmente nos grupos com dependência grave do tabaco (RIGOTTI NA, et al., 2021).

O tratamento para cessação do tabagismo adotado pelo Sistema Único de Saúde (SUS), aborda aconselhamento terapêutico associado com tratamento farmacológico e tempo de tratamento preconizado de um ano. Sendo os medicamentos disponíveis no SUS: bupropiona e nicotina tanto na forma de adesivo 
quanto goma e pastilha (MINISTÉRIO DA SAÚDE, 2020). Na avaliação inicial é importante conhecer algumas particularidades de cada paciente como, o histórico tabagista, doenças, o grau de dependência de nicotina e o estágio de motivação segundo o modelo de Prochaska e colaboradores (RIGOTTI NA, et al., 2021).

A abordagem intensiva adotada no tratamento dos fumantes deve ser coordenada por profissional de nível superior capacitado e realizada preferencialmente em grupo, mas podendo ser feita também de forma individual. O método é baseado na terapia cognitivo-comportamental e consiste em fornecer informações, estimular o autocontrole e autocuidado para que o próprio paciente seja protagonista da mudança (MINISTÉRIO DA SAÚDE, 2020).

Nas intervenções em grupo, o Instituto Nacional de Câncer sugere que os encontros tenham no máximo 15 pessoas e feitos semanalmente no primeiro mês e quinzenalmente no segundo mês totalizando seis. Com encontros de manutenção mensais, misturando os participantes dos diferentes grupos. O coordenador deve sempre ser empático acolhedor e respeitoso. A vantagem da adoção do grupo é usar a interação entre os participantes como forma de incentivo e apoio. Os objetivos dos encontros são de discutir e aprofundar em temas com a finalidade de ajudar o paciente a superar a dependência. A sequência dos encontros tem o objetivo de preparar o fumante para resolver seus problemas, estimular a autoeficácia para resistir às fissuras, prepará-lo para prevenção de recaídas e criar estratégias para lidar com o estresse (ANTUNES FM, et al., 2018).

Métodos como, o uso de cigarro sem droga e as Práticas Integrativas e Complementares em Saúde (PICS) como hipnoterapia, acupuntura, aromaterapia e meditação mindfulness não apresentam, até o momento, evidências científicas que comprovem sua eficácia. Não sendo recomendados como recurso isolado no tratamento do tabagismo (RIGOTTI NA, et al., 2021).

Medidas auxiliares, como os canais telefônicos são uma medida com baixo custo e alta abrangência, que aumenta a cessação do tabagismo. No Brasil o telefone 136, Disque Saúde, faz orientações sobre cessação do tabagismo. Quando comparadas a intervenção mínima/breve, materiais de autoajuda e farmacoterapia isolada, três ou mais contatos telefônicos aumentaram as chances de cessação (MINISTÉRIO DA SAÚDE, 2020). Outra estratégia possível são as intervenções de autoajuda, que envolvem a distribuição de material escrito como panfletos, livretos, manuais e material audiovisual. São fáceis de realizar e podem funcionar como complementares aos outros recursos (WHITTAKER R, et al., 2019).

A combinação de aconselhamento e tratamento medicamentoso tem eficácia superior e deve ser sempre ser usada, exceto nos casos que houver contraindicação ou em populações específicas como gestantes, tabagistas leves e adolescentes nos quais a evidência do uso ainda é insuficiente (WINDLE SB, et al., 2016). Nos casos em que o paciente tiver uma ou mais dessas características, como: ausência de sintomas de abstinências, uso igual ou menor do que 5 cigarros ao dia, fumar o primeiro cigarro mais de 01 hora após acordar ou pontuar até 4 no teste de Fagerström, o tratamento é feito preferencialmente com o aconselhamento intensivo isolado (MINISTÉRIO DA SAÚDE, 2020).

A Força-Tarefa de Saúde Preventiva dos Estados Unidos (USPSTF) segue a mesma linha do Protocolo de Diretrizes Terapêuticas do Tabagismo de 2020 e também recomenda intervenções comportamentais associada à farmacoterapia para cessação do tabagismo em adultos (USPSTF, 2021). Os fármacos usados como primeira linha são a nicotina, bupropiona e vareniclina. Um fármaco alternativo de menor custo é a nortriptilina, porém com menor evidência e mais efeitos adversos (LEMES EDO, et al., 2017).

Os fármacos disponíveis no SUS são a bupropiona com comprimidos de $150 \mathrm{mg}$ de liberação prolongada; a nicotina transdérmica de liberação lenta, na forma de adesivo com apresentações de $7 \mathrm{mg}, 14 \mathrm{mg}$ e $21 \mathrm{mg}$; e, a nicotina de liberação rápida na forma de goma de mascar de $2 \mathrm{mg}$ e pastilha de $2 \mathrm{mg}$. Lembrando que a reposição de nicotina só deve ser feita após a cessação do tabagismo e/ou do uso de derivados do tabaco (MINISTÉRIO DA SAÚDE, 2020).

Mas é importante, antes de considerar o tratamento para cessação do tabagismo, uma avaliação da saúde mental do indivíduo, visto que pacientes com história de ansiedade ou depressão podem necessitar de acompanhamento multiprofissional. Além disso, os fumantes após a cessação do tabagismo, podem ter ganho 
de peso que ocorre mais comumente nos primeiros 6 meses, ficando em torno de 4 a $5 \mathrm{~kg}$ e tende a estabilizar após o primeiro ano de cessação (RIGOTTI NA, et al., 2021).

Após o indivíduo parar de fumar, o foco deve ser na prevenção de recaídas, mais comuns nas duas primeiras semanas. Assim, na fase de cessação é importante elogiar o sucesso, o manejo das dificuldades e reforçar os benefícios da cessação. Quanto maior for o período de abstinência na última tentativa, maior a chance de sucesso na tentativa atual e é comum que os fumantes necessitem de mais de uma tentativa até realmente conseguirem parar de fumar. A prevenção de recaídas tem como objetivo estimular a autoeficácia, evitar situações gatilho e focar nos motivos que levaram o paciente a parar de fumar e a importância dele se manter abstinente (GUSSO G, et al., 2018).

\section{CONSIDERAÇÕES FINAIS}

O tabagismo está na história da humanidade e tem um grande impacto na saúde pública e na economia. Dessa forma, conhecer do assunto é relevante para todo o profissional de saúde. Sabe-se que o melhor tratamento é a combinação de medidas comportamentais e farmacológicas. Podendo ser feito de várias maneiras, sempre considerando as condições socioeconomicas do paciente, buscando um acolhimento atencioso e fácil para os usuários do sistema público de saúde. Assim, entender o básico dessa abordagem ajuda no manejo dos pacientes tabagistas e evita os desdobramentos ruins do tabagismo.

\section{REFERÊNCIAS}

1. ALQAHTANI JS, et al. Prevalence, Severity and Mortality associated with COPD and Smoking in patients with COVID19: A Rapid Systematic Review and Meta-Analysis. PLoS ONE, 2020; 15(5): e0233147.

2. ANTUNES FM, et al. Revisão Bibliográfica Sistemática de Grupo para a Cessação do Tabagismo-sob a Abordagem Cognitivo-Comportamental. Trabalho de Conclusão de Residência (Residência Multiprofissional Atenção em Saúde Mental). Universidade Federal de Uberlândia, Uberlândia, 2018, 22p.

3. GUSSO G, et al. Tratado de Medicina de Família e omunidade 2.Ed. Porto Alegre: Artmed, 2018; 2083-2091p.

4. INSTITUTO NACIONAL DO CÂNCER (INCA). Dados e números da prevalência do tabagismo. 2021a. Disponível em: https://www.inca.gov.br/observatorio-da-politica-nacional-de-controle-do-tabaco/dado s-e-numeros-prevalenciatabagismo. Acesso em: 1 de out. de 2021.

5. INSTITUTO NACIONAL DO CÂNCER (INCA). Produção de fumo e derivados. 2020. Disponível em: $\mathrm{https://www.inca.gov.br/observatorio-da-politica-nacional-de-controle-do-tabaco/producao-fumo-e-derivados.} \mathrm{Acesso}$ em: 1 de out. de 2021.

6. INSTITUTO NACIONAL DO CÂNCER (INCA). O controle do tabaco no Brasil: uma trajetória. 2012. Disponível em: https://bvsms.saude.gov.br/bvs/publicacoes/exposicao_controle_tabaco_brasil_trajet oria.pdf. Acesso em: 1 de out. de 2021.

7. INSTITUTO NACIONAL DO CÂNCER (INCA). Custos atribuíveis ao tabagismo. 2021b. Disponível em: https://www.inca.gov.br/observatorio-da-politica-nacional-de-controle-do-tabaco/custos-atribuiveis-ao-tabagismo.

Acesso em: 1 de out. de 2021.

8. INSTITUTO NACIONAL DO CÂNCER (INCA). Doenças relacionadas ao tabagismo. 2021c. Disponível em: https://www.inca.gov.br/observatorio-da-politica-nacional-de-controle-do-tabaco/doencas-relacionadas-aotabagismo. Acesso em: 1 de out. de 2021.

9. INSTITUTO NACIONAL DO CÂNCER (INCA). Tabagismo e coronavirus. 2021d. Disponível em: https://www.inca.gov.br/programa-nacional-de-controle-do-tabagismo/tabagismo-e-coronavirus. Acesso em: 1 de out. de 2021.

10. INSTITUTO NACIONAL DO CÂNCER (INCA). Abordagem breve/mínima/básica na cessação do tabagismo. 2021 . Disponível em: https://www.inca.gov.br/sites/ufu.sti.inca.local/files//media/ document//abordagem_tabagismo_web.pdf. Acesso em: 1 de out. de 2021.

11. LEMES EDO, et al. Análise do programa nacional de controle do tabagismo do ministério da saúde. Uniciências, 2017; 21(2): 86-92.

12. LOPES FM, SILVEIRA K. Efeito de estratégias e técnicas cognitivo-comportamentais no tratamento do tabagismo. Revista Brasileira de Terapias Cognitivas, 2020; 16(1): 59-66.

13. MACIEL RDR, et al. Grau de dependência à nicotina de pacientes atendidos para tratamento do tabagismo em universidade pública. SMAD Revista Eletrônica Saúde Mental Álcool e Drogas, 2021; 17(1): 48-57.

14. MINISTÉRIO DA SAÚDE. Protocolo Clínico e Diretrizes Terapêuticas do Tabagismo. Portaria Conjunta №10, 16 abril de 2020. Disponível em: https:/www.in.gov.br/en/web/dou/-/portaria-conjunta-n-10-de-16-de-abril-de-2020253756566. Acesso em: 14 de out. de 2021.

15. ORGANIZAÇÃO MUNDIAL DE SAÚDE (OMS). Regional Office for the Eastern Mediterranean. Eastern Mediterranean Health Journal. East Mediterr Health J. janeiro de, 2020; 26(1): 4-133 
16. PORTES LH, et al. Trajetória da política de controle do tabaco no Brasil de 1986 a 2016. Cadernos de Saúde Pública, 2018; 34(2): e00017317.

17. RIGOTTI NA, et al. Overview of smoking cessation management in adults. UpToDate, 2021. Disponível em: https://www.uptodate.com/contents/pharmacotherapy-for-smoking-cessation-in-adults. Acesso em: 14 de out. de 2021.

18. RIGOTTI NA, et al. Benefits and consequences of smoking cessation. UpToDate, 2021. Disponível em: https://www.uptodate.com/contents/benefits-and-consequences-of-smoking-cessation. Acesso em: 14 de out. 2021.

19. ROCHA SAV, et al. Prevalência de tabagismo e motivos para continuar a fumar: estudo de base populacional. Jornal Brasileiro de Pneumologia, 2019; 2019; 45(4): e20170080

20. SANTOS MDV, et al. Prevalência de estratégias para cessação do uso do tabaco na Atenção Primária à Saúde: uma revisão integrativa. Ciência \& Saúde Coletiva, 2019; 24(2): 563-572

21. SINDICATO INTERESTADUAL DA INDÚSTRIA DO TABACO (SINDITABACO). Tabaco: Relevância Econômica e Social. 2019. Disponível em: https:/www.gov.br/agricultura/pt-br/assuntos/camaras-setoriaistematicas/documentos/camaras-setoriais/tabaco/2019/sinditabaco-relatorio-institucional-2019.pdf/view. Acesso em: 1 de out. de 2021.

22. SZKLO AS, BERTONI N. Relação entre a epidemia de tabagismo e a epidemia recente de Covid-19: um panorama atual das evidências científicas. Revista Brasileira de Cancerologia Cancerologia, 2020. Disponível em: https://rbc.inca.gov.br/revista/index.php/revista/article/view/1105. Acesso: 14 de out. de 2021.

23. US PREVENTIVE SERVICES TASK FORCE (USPSTF). Interventions for Tobacco Smoking Cessation in Adults, Including Pregnant Persons: US Preventive Services Task Force Recommendation Statement. JAMA, 2021; 325(3): 265-279.

24. WINDLE SB, et al. Combination Therapies for Smoking Cessation: A Hierarchical Bayesian Meta-Analysis. American Journal of Preventive Medicine, 2016; 51(6): 1060-1071.

25. WHITTAKER R, et al. Mobile phone text messaging and app-based interventions for smoking cessation. Cochrane Database of Systematic Reviews, 2019; 10(10): CD006611. 